\title{
The Astro-WISE optical image pipeline
}

\section{Development and implementation}

\author{
John P. McFarland • Gijs Verdoes-Kleijn • \\ Gert Sikkema • Ewout M. Helmich • \\ Danny R. Boxhoorn • Edwin A. Valentijn
}

Received: 5 October 2011 / Accepted: 10 October 2011 / Published online: 25 November 2011

(C) The Author(s) 2011. This article is published with open access at Springerlink.com

\begin{abstract}
We have designed and implemented a novel way to process widefield astronomical data within a distributed environment of hardware resources and humanpower. The system is characterized by integration of archiving, calibration, and post-calibration analysis of data from raw, through intermediate, to final data products. It is a true integration thanks to complete linking of data lineage from the final catalogs back to the raw data. This paper describes the pipeline processing of optical wide-field astronomical data from the WFI (http:/www.eso.org/lasilla/instruments/wfi/) and OmegaCAM (http://www.astro-wise.org/ omegacam/) instruments using the Astro-WISE information system (the Astro-WISE Environment or simply AWE). This information system is an environment of hardware resources and humanpower distributed over Europe. AWE is characterized by integration of archiving, data calibration, post-calibration analysis, and archiving of raw, intermediate, and final data products. The true integration enables a complete data processing cycle from the raw data up to the publication of science-ready catalogs. The advantages of this system for very large datasets are in the areas of: survey operations management, quality control, calibration analyses, and massive processing.
\end{abstract}

Keywords Wide-field imaging • Data processing • Information system

J. P. McFarland ( $\varangle) \cdot$ G. Verdoes-Kleijn · G. Sikkema .

E. M. Helmich · D. R. Boxhoorn · E. A. Valentijn

Kapteyn Astronomical Institute, P.O. Box 800,

9700 AV Groningen, The Netherlands

e-mail: mcfarland@astro.rug.nl 


\section{Introduction}

The rapid increase in the number of astronomical data sets and even faster increase of overall data volume demands a new paradigm for the scientific exploitation of optical and near-infrared imaging surveys. Historical surveys have been digitized (POSS and its southern counterpart) or are in the process of being digitized. ${ }^{1}$ In recent years surveys have been performed which cover hundreds or thousands of square degrees up to the whole sky (SDSS, 2MASS, CFHTLS, etc.). Many more are in progress or coming up with increasing spatial resolution, depth, and survey areas (OmegaCAM on VST, VIRCAM on VISTA, Pan-STARRS, LSST, etc.). The data rate of existing surveys is rapidly approaching terabytes per night, leading to survey volumes well into the petabyte regime and the new surveys will add many tens of petabytes to this. ${ }^{2}$ Hundreds of terabytes of data will start entering the system when ESO's OmegaCAM camera starts operations in Chile in late-2011. Several large surveys plan to use the Astro-WISE information system to manage their data: the $1,500 \mathrm{deg}^{2}$ KIDS Survey, ${ }^{3}$ the Vesuvio Survey ${ }^{4}$ of nearby superclusters, the OmegaWhite ${ }^{5}$ white dwarf binary survey and the OmegaTrans ${ }^{6}$ search for transiting variables.

Quality control is typically one of the largest challenges in the chain from raw data of the "sensor networks" to scientific papers. It requires an environment in which all non-manual qualification is automated and the scientist can graphically inspect where needed by easily going back and forth through the data (the pixels) and metadata (everything else) of the whole processing chain for large numbers of data products. The full quality control mechanisms are treated in complete detail in the Astro-WISE Quality Control paper [4].

The really novel aspect of this new paradigm is the long-term preservation of the raw data and the ability of re-calibrating it to the requirements of new science cases. The data of the majority of these surveys is fully public: any astronomer is entitled to a copy of the data. ${ }^{7}$ Therefore the same survey data is used for not only science cases within the original plan, but many new science cases the original designers of the survey were not planning to do themselves or did not foresee. To be able to do this successfully requires that everyone is provided access to detailed information on the existing calibration procedures and resulting quality of the data at every stage of the processing, that is, have

\footnotetext{
${ }^{1}$ See, e.g., http://archive.stsci.edu/dss/, http://tdc-www.harvard.edu/plates/, http://www.lsw.uniheidelberg.de/projects/scanproject/

${ }^{2}$ See, e.g., http://www.lsst.org/lsst/science/technology, http://pan-starrs.ifa.hawaii.edu/public/designfeatures/data-handling.html

${ }^{3} \mathrm{http}: / /$ www.astro-wise.org/projects/KIDS/

${ }^{4} \mathrm{http}: / /$ www.astro-wise.org/projects/VESUVIO/

${ }^{5} \mathrm{http} / / /$ www.astro-wise.org/projects/OMEGAWHITE/

${ }^{6} \mathrm{http} / /$ www.astro-wise.org/projects/OMEGATRANS/

${ }^{7}$ See, e.g., http://www.eso.org/sci/observing/policies/PublicSurveys.html
} 
access to the data and the metadata, including process configuration at every step in the chain from raw data to final data products.

In this paper we describe the reduction of data in the Astro-WISE information system, generally referred to as the Astro-WISE Environment (hereafter $A W E$ ). The processing of data from both the WFI and OmegaCAM instruments has been used to qualify the pipeline, the results of which have been or will be included in separate publications, for example [7,8]. The remainder of this section briefly describes some key concepts of AWE covered in detail elsewhere: previously in Valentijn et al. [6] and more currently in Begeman et al. [1]. Sections 2 and 3 describe how an instrument is calibrated and how science data is processed. Finally, Section 4 presents the summary.

\subsection{Context}

Context is the primary tool of project managers in AWE. Each process target (i.e., the result of some processing step, see Section 1.2.2) in AWE is created at a specific privilege level. Privilege levels are analogous to the permission levels of a Unix/Linux file system (e.g., privilege levels 1, 2, 3 map loosely to permission levels user, group, other). To allow access to their desired set of objects, users can set their privilege level and their project.

This concept of context is completely about visibility of the objects in AWE and nothing else. Proprietary data is protected from access by all but authorized users and undesirable data can be hidden for any purpose (e.g., to use project-specific calibrations instead of general ones). All processing is done within this framework, allowing complete control over what is processed and how, and how it is published between project groups and to the world.

Visibility for processing targets is not only governed by the privilege level, but also by validity. Three properties dictate validity:

1. is_valid-manual validity flag

2. quality_flags-automatic validity flag

3. timestamps - validity ranges in time (for calibrations only)

Determining what needs to be processed and how is indicated by setting any or all of the above flags. For instance, obviously poor quality data can be flagged by setting its is_valid flag to 0 , preventing it from ever being processed automatically. The calibrations used are determined by their timestamps (Which calibrations are valid for the given data?) and the quality of processed data by the automatic setting of its quality_flag (Is the given data good enough?). Good quality data can then be flagged for promotion (is_valid $>1$ ) and eventually promoted in privilege by its creator (published from level 1 to 2 ) so it can be seen by the project manager who will decide if it is worthy to be promoted once again (published from level 2 to 3 or higher) to be seen by the greater community. 


\subsection{Provenance: full dependency linking}

AWE uses its federated database to link all data products to their progenitors (dependencies), creating a full data lineage of the entire processing chain. This allows creation of complete data provenance for any data item in the system at any time.

\subsubsection{Full data lineage}

Raw data is linked to the final data product via database links within the data object, allowing all information about any piece of data to be accessed instantly. See Mwbaze et al. [5] for a detailed description of the AWE's data lineage implementation. This data linking uses the power of Object-Oriented Programming to create this framework in a natural and transparent way.

\subsubsection{Object-oriented data model}

AWE uses the advantages of Object-Oriented Programming (OOP) to process data in the simplest and most powerful ways. In essence, it turns the aforementioned data objects into OOP objects, called process targets (or ProcessTargets), that are instances of classes with attributes and methods that can be inherited (see Figs. 1 and 2 for an overview of an Astro-WISE object model). Each of these ProcessTarget instances knows of all of its local and linked metadata, and knows how to process itself. Each persistent attribute of an object is linked to metadata or to another object that itself contains links to its own metadata.

The code for AWE is written in Python, a programming language highly suitable for OOP. Consequently, Python classes are associated with the various conventional calibration images, data images, and other derived data products. For example, in AWE, bias exposures become instances of the RawBiasFrame class, and twilight (sky) flats become instances of the RawTwilightFlatFrame class. These instances of classes are the "objects" of OOP.

For the remainder of this document, the class names of objects, their properties, and methods will be in teletype font for more clear identification.

\subsection{Target-based processing}

The most unique aspect of AWE is its ability to process data based on the final desired result to an arbitrary depth. In other words, the data is pulled from the system by the user. The desired result is the target to be processed, and the framework used is called target processing. Target processing uses methods similar to those found in the Unix/Linux make utility. When a target is requested, its dependencies are checked to see if they are up-to-date. If there is a newer dependency or if the requested target does not exist, the target is (re)made. This process is recursive and is an example of backward chaining. 


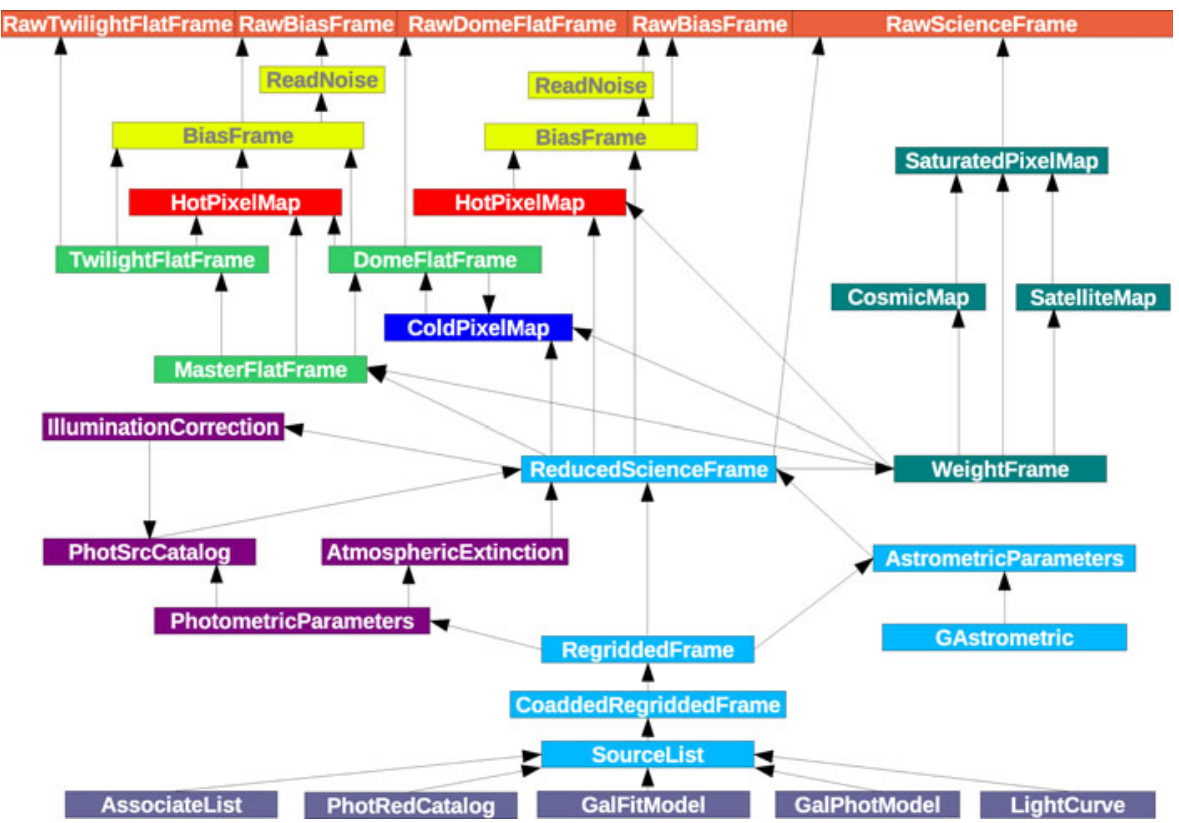

Fig. 1 A target diagram: slightly simplified object model that is a view of the dependencies of "targets" to raw observational data. The arrows indicate the backward chaining to the raw data, not the progression through any processing pipeline. The colors provide a visual grouping of similar types of data products

\subsubsection{Backward chaining}

At the base of AWE target processing is the concept of backward chaining. Contrary to the typical case of forward chaining (e.g., ob ject $N$ is processed into ob ject $\mathrm{N}+1$ is processed into ob ject $\mathrm{N}+2$, etc.). AWE database links allow the dependency chain to be examined from the intended target (even if it does not yet exist) all the way back to the raw data. The above scenario would then look like: if targetM is up-to-date, check if targetM-1 is up-to-date; if targetM-1 is up-to-date, check if targetM-2 is up-to-date; etc., processing as necessary until targetM (and all targets it depends on) exists and is upto-date. ${ }^{8}$ This is the AWE implementation of backward chaining that is used in target processing (see Fig. 1 for an example with astronomical data).

\subsubsection{Processing parameters}

As mentioned earlier, conventional astronomical calibration images/products as well as science products are collectively referred to as process targets and

\footnotetext{
${ }^{8}$ Note that the counting of targets is reversed in the backward chaining example, as this is the direction in which the up-to-date check is run.
} 
Fig. 2 A Astro-WISE

hierarchical object model. A simplified object model of the target classes shown in Fig. 1 illustrating their inheritance relationship to each other. The classes without color do not appear in the previous figure, but are nonetheless part of the hierarchy and are shown for clarity. Every target inherits from DBObject (a database object), but only those with associated bulk data (typically a file stored on a dataserver) inherit from DataObject

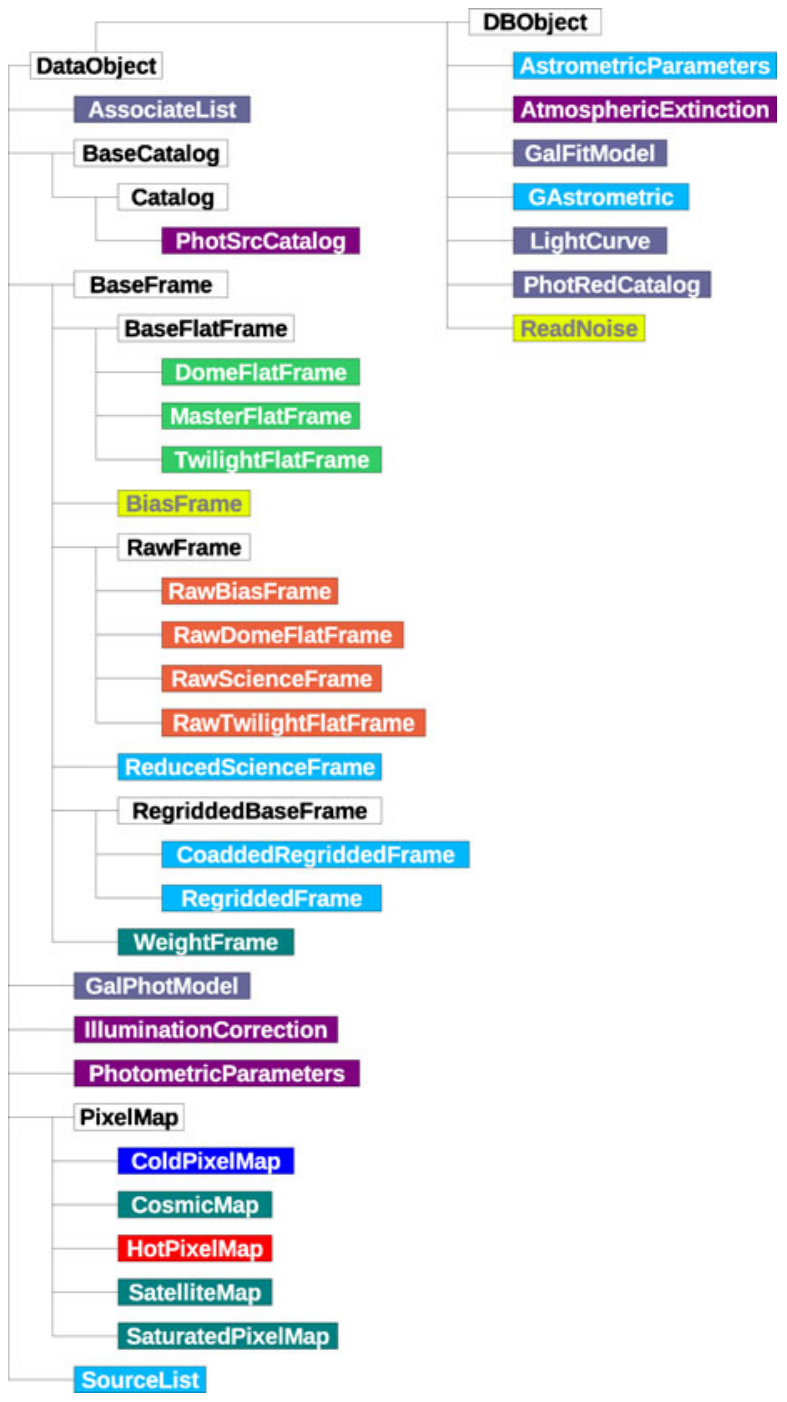

inherit from the Process Target class. Each ProcessTarget has an associated processing parameters object, an instance of a class named after the respective process target class (e.g., SomeTarget. SomeTargetParameters) which stores configurable parameters that guide the processing or reprocessing of that target. Those ProcessTargets that use external programs in their derivation may have additional objects associated with them which contain the configuration of the external program that was used.

These processing parameters are stored in an object linked to the Processtarget for comparison by the system and to allow the all persons 
involved in survey operations to discover which settings resulted in the best data reduction.

\subsection{On-demand reprocessing}

AWE combines all of the above concepts into a coherent archiving and processing system. All the information about a particular instrument and its calibration and processing history is stored in the federated database within the object-oriented data model with full linking of the data lineage. The values of the process parameters of all objects in the dependency chain and all the results of the integrated (and manual) quality controls of the target of interest (regardless of visibility or existence) are used to determine if that target can or should be (re)built and how. This data pulling is the heart of AWE and is called target processing (see Fig. 3 and http://process.astro-wise.org/).

\subsubsection{Raw data sacred}

As mentioned earlier, AWE does not provide as the ultimate end of the processing chain a static data release. The system allows for survey data to be reprocessed for any reason and for any purpose. If a newer, better calibration is made, or if a different purpose requires a different processing technique, the

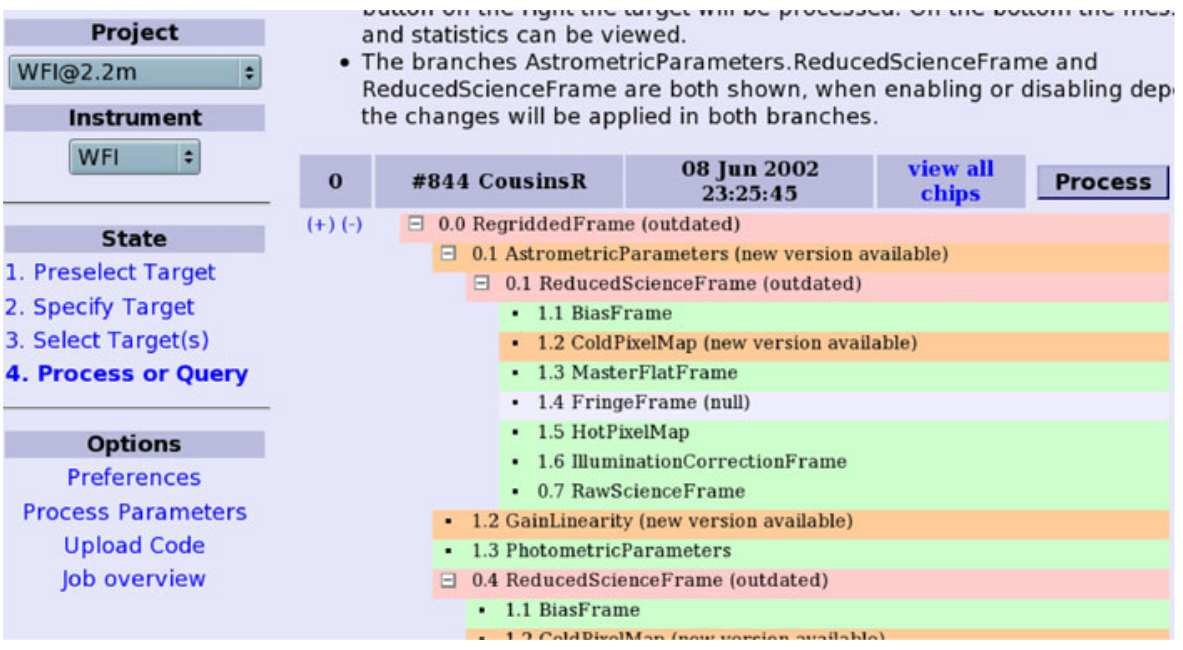

Fig. 3 A screen-capture of part of the web-based target processing interface. On the left are high-level processing settings (e.g., project, processing step, options). On the right is the result of the query for a particular target. Green rows show dependencies that are ready and will not be processed, red and orange rows show dependencies that are either outdated (need to be rebuilt) or already have a new version available. This section is a glimpse at the information used to dynamically construct the workflow that will create the eventual processing pipeline. Only those targets in the red rows will actually be processed 
data can be easily reprocessed. This is only possible when the raw survey data is retained in its original form. In AWE raw data is always preserved.

\subsubsection{On-the-fly (re)processing}

Target processing does not use static information to determine what gets processed how. As seen in all the previous sections, all the survey data, its dependency linkages and processing parameters are all reviewed to allow any target to be (re)processed on-demand as needed. All these dependencies create a built-in workflow, automatically processing only those targets that need it. This on-the-fly (re)processing is the hallmark of the AWE information system.

\section{Calibration pipeline: correcting the pixels}

The philosophy of $A W E$ is to share improved insight in calibrations. In $A W E$, calibration scientists can, over time, have many versions of calibration results at their disposal. From this they determine (subtle) long term trends in instrument, telescope and atmospheric behaviour and can collaborate to improve the calibration procedures for that instrument in AWE accordingly. The complete observational system (generally termed "the instrument" for simplicity) eventually becomes calibrated over its full operational period as opposed to a series of individual nights calibrated from data in a limited time window. Figure 4 shows the schematic view of the pixel calibrations pipeline. This gives an overview of the flow of the pixel calibrations to be described in the coming sections. It is continued in the photometric pipeline schematic in Fig. 5.

In the $A W E$, calibration objects have a set validity range in time or per frame object that depends upon the calibration object (the defaults are specified per calibration object in Table 1 below). The default validity time range

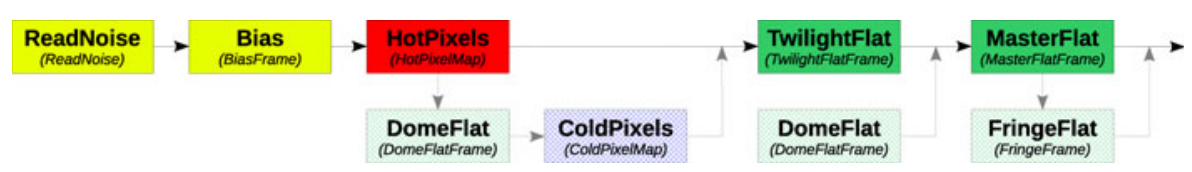

Fig. 4 Schematic flow of the pixel calibrations pipeline following the coloring in Fig. 1. The recipes, also called Tasks, used to produce various ProcessTargets are indicated in each box (with their data product in parentheses) and described in the various sections. The arrows connecting them indicate the direction of processing. Note that the sections with the hatched boxes are optional branches in this pipeline, and the arrow at the end leads to the beginning of the photometric pipeline schematic in Fig. 5. Also note, in order to simplify this diagram, the GainLinearity, DarkCurrent and NightSkyFlatFrame objects have been omitted 


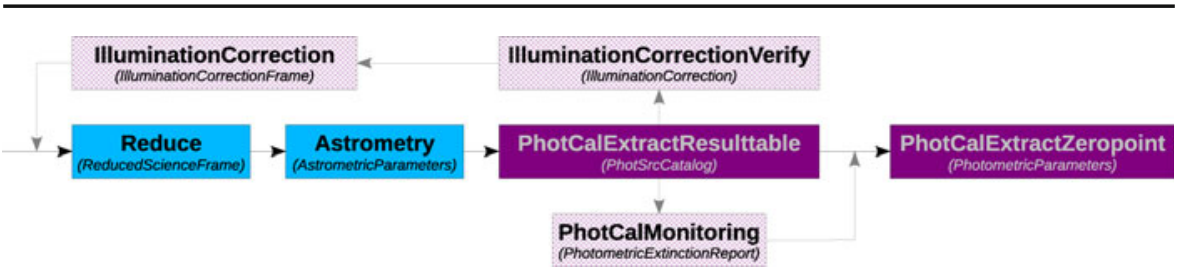

Fig. 5 Schematic flow of the photometric pipeline following the coloring in Fig. 1. The recipes, also called Tasks, used to produce various ProcessTargets are indicated in each box (with their data product in parentheses) and described in the various sections. The arrows connecting them indicate the direction of processing. Note that the sections with the hatched boxes are optional branches in this pipeline, and the input follows from the pixel calibrations pipeline shown in Fig. 4

(timestamp_start to timestamp_end) can be altered on the commandline using context methods (see Section 1.1), or via the CalTS web-service (see Fig. 6).

Be sure to note that, with the exception of parts of the astrometric calibration derivation and most of the photometric calibration derivation, all calibration objects are normally processed in a parallel environment, one detector chip per CPU node.

Many Process Target's have configurable processing parameters to control how they are processed. Table 2 gives an overview of these process_ params for the calibration pipeline. In addition to the process_params associated directly with the ProcessTarget, there exist object representations of configuration files for external programs wrapped in Python (e.g., SExtractor, SWarp, etc.).

Table 1 Default validities of calibration ProcessTargets

All time spans are centered on local midnight of the day the source observations were taken unless otherwise indicated

\begin{tabular}{ll}
\hline ProcessTarget & Default validity \\
\hline GainLinearity & 1 day \\
ReadNoise & 1 day \\
BiasFrame & 1 day \\
DarkCurrent & 1 day \\
HotPixelMap & Same as source BiasFrame \\
ColdPixelMap & Same as source FlatFrame \\
DomeFlatFrame & 7 days \\
TwilightFlatFrame & 7 days \\
NightSkyFlatFrame & 1 day \\
MasterFlatFrame & 7 days \\
FringeFrame & 1 day \\
AstrometricParameters & Points to one frame only \\
AtmosphericExtinctionCurve & 1 day \\
PhotometricReport & 1 day \\
PhotometricParameters & 1 day \\
IlluminationCorrection & 1 day \\
IlluminationCorrectionFrame & Same as source \\
& IlluminationCorrection \\
\hline
\end{tabular}




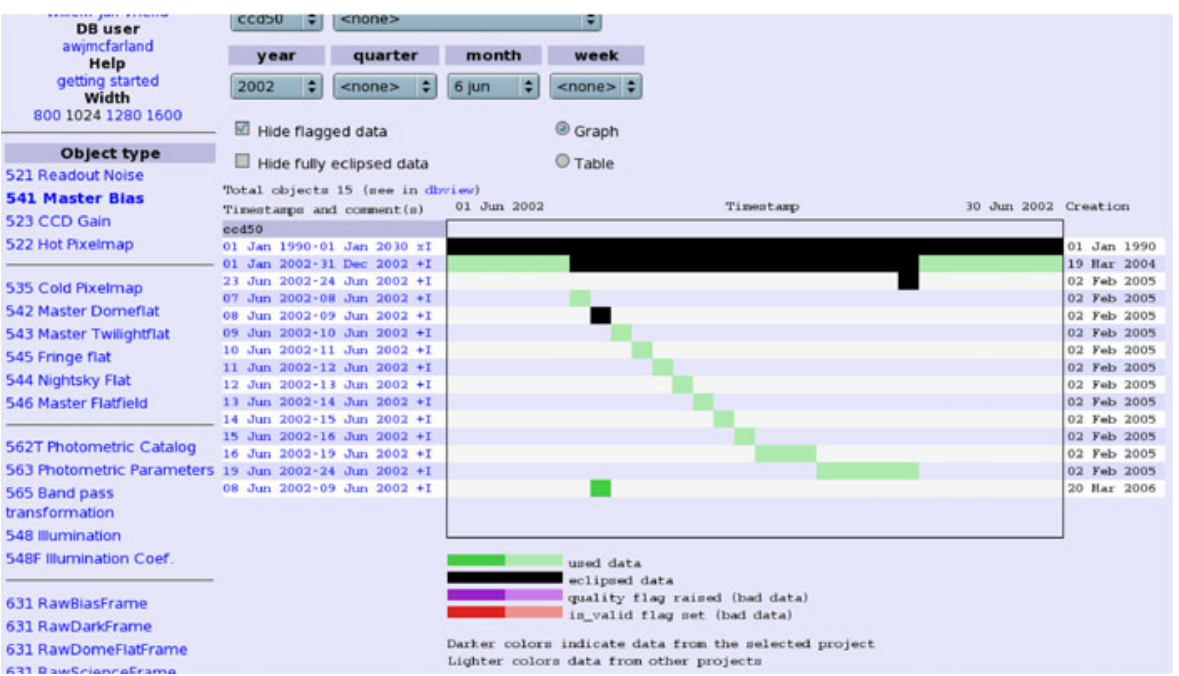

Fig. 6 A screen-capture of CalTS, the web-based Calibration TimeStamp service. The purpose of this service is to give a graphical representation of the temporal validity ranges of calibration objects in AWE. On the left can be selected the ProcessTarget of interest, at the top are some of the query criteria, and below this, the graphical validity of the Process Target. Colored bars indicate the most recent valid objects (objects flagged invalid are hidden), while black bars indicate where objects are "eclipsed" by newer calibrations. It is always assumed that the newest valid ProcessTarget is the best and this will be the one used during processing. The timestamps and validity can be modified by an interface raised by clicking on the date range for a given object. http://calts.astro-wise.org/

\subsection{ReadNoise}

The read-out noise is the noise introduced in the data by the read-out process of detector chips. It is measured from pairs of bias exposures. The RMS scatter of the differences between two bias exposures is computed. The read noise in ADU is determined via division of this value by $\sqrt{2}$. The read noise value is stored in the database using the ReadNoise class.

\subsection{GainLinearity}

The gain is the conversion factor between the signal in ADU's supplied by the readout electronics and the detected number of photons (in units $\mathrm{e}^{-}$/ADU). For OmegaCAM, a procedure (template) to determine the gain (and the linearity of the detector chips) is defined that involves taking two series of 10 dome flatfield exposures with a wide range of exposure times, and deriving the RMS of the differences of two exposures taken with similar exposure (integration time). The regression of the square of these values with the median level yields the conversion factor in $\mathrm{e}^{-} / \mathrm{ADU}$ (assuming noise dominated by photon shot noise). A linear fit of exptimes vs. median_sum gives a measure of the linearity. For most instruments default gain values 
Table 2 Processing parameters and their generic default values

\begin{tabular}{|c|c|c|c|}
\hline Class & process_param & Value & $\overline{\text { Units }}$ \\
\hline \multirow[t]{2}{*}{ ReadNoise } & rejection_threshold & 5.0 & \\
\hline & maximum_iterations & 5 & \\
\hline \multirow[t]{3}{*}{ GainLinearity } & overscan_correction & 6 & \\
\hline & rejection_threshold & 5.0 & \\
\hline & maximum_iterations & 5 & \\
\hline \multirow[t]{2}{*}{ BiasFrame } & overscan_correction & 6 & \\
\hline & sigma_clip & 3.0 & \\
\hline \multirow[t]{2}{*}{ HotPixelMap } & rejection_threshold & 5.0 & \\
\hline & maximum_iterations & 5 & \\
\hline \multirow[t]{2}{*}{ ColdPixelMap } & threshold_low & 0.94 & \\
\hline & threshold_high & 1.06 & \\
\hline \multirow[t]{2}{*}{ DomeF latFrame } & overscan_correction & 6 & \\
\hline & sigma_clip & 3.0 & \\
\hline \multirow[t]{2}{*}{ TwilightFlatFrame } & overscan_correction & 6 & \\
\hline & sigma_clip & 3.0 & \\
\hline \multirow[t]{5}{*}{ MasterFlatFrame } & dig_filter_size & 9.0 & \\
\hline & mirror_xpix & 75 & Pixel \\
\hline & mirror_ypix & 150 & Pixel \\
\hline & median_filter_size & 36 & Pixel \\
\hline & combine_type & 1 & \\
\hline \multirow[t]{2}{*}{ PhotometricParameters } & sigclip_level & 1.5 & \\
\hline & min_nmbr_of_stars & 3 & \\
\hline
\end{tabular}

These values are representative of the typical value for any instrument. Some instruments may have values that different from these based on experience with that instrument. See the document page linked from the class name or appropriate links on http://doc.astro-wise.org/astro.main.html for more details

have been determined or taken from the literature and are in the system, so it is usually not necessary to make new values for them. If this is desired, a specialized dataset similar to that described must be used. The class used to store the gain in the database is the GainLinearity.

\subsection{BiasFrame}

The signal in raw scientific frames contains a component that is due to a bias current introduced by the AD converter on a FIERA ${ }^{9}$ or other detector controller. This component shows up as an offset to the signal. In most CCD detectors, the bias-offset has the following characteristics: i) the bias level grows to its asymptotic level in the first few hundred lines, and ii) the bias level depends on the total signal in a given line. Therefore, an initial bias correctionthe overscan correction, is applied when the overscan region exists (cheaper

\footnotetext{
${ }^{9}$ Acronym for Fast Imager Electronic Readout Assembly CCD controller, http://www.eso.org/ projects/odt/Fiera/
} 
CCDs and IR detectors tend not to have these regions). The method used is one of a set of methods ranging from no correction, to subtraction of a constant value derived from one of the prescan or overscan regions, to subtracting an average value per column or row, smoothed or not, to hybrid corrections for complex geometries. Each of these methods is given an index which is stored in the database, constituting the only really "free" parameter in the system.

In addition, the bias offset exhibits a residual pattern, which is measured by the master bias frame, an instance of the Biasframe class. To construct the master bias, a series of $N$ (usually 5-10) zero-second bias exposures is overscan-corrected and averaged, rejecting $5 \sigma$ outliers $(\sigma=$ readout noise from a ReadNoise object), due to particle hits during read-out. The resulting master bias frames will be used for the correction of all frames.

As the read-out noise dominates the RMS scatter in the bias frames, while the shot noise of the sky background dominates the RMS scatter on the sky images, which is nominally much larger than the readout noise, it is sufficient to characterize the bias value at individual pixels with an accuracy of (readout noise $/ \sqrt{N})$.

\subsection{DarkCurrent}

In AWE, no formal dark frame subtraction is performed. Current, liquid nitrogen cooled instruments tend to have little or no appreciable two-dimensional dark current structure, any of which will normally be removed with the sky background. As AWE was created explicitly for such an instrument, dark frame correction was not included. There is, however, some treatment of this effect through the DarkCurrent class. The purpose of this class is to determine the total dark current and the particle event rate of a detector chip. This is not used for calibration, but for the detector chain health.

The dark current, excess signal due to heat in a detector chip, is measured by taking three identically timed exposures (typically $1 \mathrm{~h}$ ) with the camera shutter closed. The resulting frames are trimmed, overscan- and bias-corrected, then a median is taken along the $Z$-axis of the exposure stack. After iterative outlier rejection, the average value of all the pixels is the dark current in units of ADU/pixel/hour.

The same trimmed, overscan- and bias-corrected frames are used to determine the particle even rate. The source extraction software SExtractor ${ }^{10}$ is used on each image in turn to detect the number of cosmic ray particle events. A HotPixelMap can optionally be used to mask detected hot pixels. The particle event rate is determined in units of particles $/ \mathrm{cm}^{2} / \mathrm{h}$.

\footnotetext{
${ }^{10} \mathrm{http}: / /$ astromatic.iap.fr/software/sextractor/
} 


\subsection{HotPixelMap and ColdPixelMap}

Hot pixels are pixels which have high count rates despite not being illuminated. In AWE, these pixels are detected from bias images (which have an exposure time of $0 \mathrm{~s}$ ). More precisely: greater than $5 \sigma$ outliers in bias are defined as hot pixels. Cold pixels are broken pixels which have low or zero counts even when illuminated. These pixels are determined from dome flat-field exposures because those have the most uniform and consistently high counts required. Twilight flat-fields can be used if no dome flat-fields are available. In AWE, all pixels that deviate substantially, i.e., more than $4 \%$ of its surroundings, from the other pixels in the flat-field are considered cold even though brighter pixels are also detected. All deviant pixels are flagged in weight maps, a mask image, where good pixels have a value of 1 and bad pixels a value of 0 .

The procedure to create a HotPixelMap starts with calculating a background map of the master bias frame and subtracting it. This is done to avoid detecting induced charge structures and other continuous structures as hot pixels. Outliers in the background-subtracted master bias frame are bad/hot pixels. A HotPixelMap is created using the threshold determined from iterative statistics estimates. The number of hot pixels is noted as a quality control value.

The procedure to create a ColdPixelMap starts with smoothing the flatfield image. The smoothed flat is used to normalize, or "flatten" the flat to eliminate large deviations from flatness that could erroneously cause entire regions to be marked as "cold". In this flat-field image, pixels that are outside a given range $( \pm 4 \%)$ are taken to be cold pixels. Note that this invalidates any pixel whose gain differs significantly from its immediate neighbors. In particular, this also identifies pixels that are bright relative to their neighbors as "cold". Note, that pixels above the threshold are formally not cold, but are flagged anyway. In the end, HotPixelMaps and ColdPixelMaps are combined into weights of the detrended science images. A ColdPixelMap is created using the thresholds given above. The number of cold pixels is noted as a quality control value.

We use SExtractor to produce the smoothed images. SExtractor uses a robust algorithm to estimate the background on a grid and interpolate between these grid points. By measuring this background for the bias and flat-field we essentially have a fast smoothing algorithm with a large kernel, that is relatively insensitive to bad pixels.

\subsection{Flat fielding}

A flat-field is the response of the telescope-camera system to a source of uniform radiation. In $A W E$, there are different ways to construct a flat field. Dome flat-fields are created by pointing the telescope at a screen on the 
inside of the dome which is illuminated by lamps. Dome flat fields have the advantage (over twilight flat fields) that it is easy to repeatedly obtain a high signal to noise level. Disadvantages are that the direction in which light enters the telescope may be different than during night time observations, that the color of the dome lamp differs from the color of the night sky and that it is very difficult to illuminate a screen in such a way that it is a source of uniform radiation. A dome flat field is useful for tracing small scale structure variations. A disadvantage for twilight flats is that they can already contain objects like stars during exposures, which should be corrected for by dithering the twilight flats. Twilight flat fields thus are better in tracing large scale structure variations. These considerations result in the desire to combine dome flats and twilight flats by spatially filtering the two types of flat fields.

\subsubsection{DomeFlatFrame}

A DomeFlatFrame is obtained through an average with sigma rejection procedure on a stack of raw dome flats, intended to reduce photon shot noise and remove cosmic rays.

The procedure to make a DomeFlatFrame starts with 5-10 overscan corrected, trimmed and debiased raw dome flats. These are normalized to the median, taking into account hot and cold pixels, and averaged rejecting $5 \sigma$ outliers: the median in $Z$-axis of the stack is used to determine the $\sigma$ levels. The computed mean in the $Z$-axis of the stack is the final DomeFlatFrame image. Lastly, sub-window image statistics are determined for quality control purposes.

\subsubsection{TwilightFlatFrame}

A TwilightFlatFrame is obtained through an average with sigma rejection procedure on a stack of raw twilight flats, intended to remove any contamination (including stars) present on individual raw twilight flats and reduce photon shot noise.

The procedure to make a TwilightFlatFrame starts with 5-10 overscancorrected, trimmed and debiased raw dome flats. These are normalized to the median, taking into account hot and cold pixels, and averaged rejecting $5 \sigma$ outliers: the median in $Z$-axis of the stack is used to determine the $\sigma$ levels. The computed mean in the $Z$-axis of the stack is the final TwilightFlatFrame image. Lastly, sub-window image statistics are determined for quality control purposes.

\subsubsection{NightSkyFlatFrame}

Raw science images have a non-flat background, attributed to flat-field effects. Information about how to flat-field science images therefore is present in the science images themselves. The flat-field that most closely reproduces the actual gain variations of the these images can be obtained by averaging a 
large number of flat-fielded science and standard observations, taking care of properly masking the contaminating objects. Such a night-sky flat could, in principle, improve on the quality of the twilight flat and may also be suitable for fringe removal.

The procedure to create a Night SkyF latFrame starts with a minimum of 5 non-cospatial science images within a given night in a given band to achieve optimal results. Images are overscan-corrected, trimmed, debiased, flat-fielded and normalized, then stacked and a median along the $Z$-axis is calculated. This median is intended to remove any exposure-specific effects (objects, cosmic rays, satellite tracks, etc.). The median image is then normalized to the mean taking into account hot and cold pixels.

\subsubsection{MasterFlatFrame}

In AWE, a MasterFlatFrame constructed from a DomeFlatFrame (used to measure the small-scale pixel-to-pixel variation) and a Twi lightF latFrame (used to measure the large-scale variation). These spatial frequencies are separated using a Fourier technique. Night SkyF latFrames are created from raw science or standard data that has been flat-fielded with this master flatfield and can be used to improve the quality of it. This (improved) master flat-field is then used to flat-field the science and standard images in the image pipeline.

In practice, not all three flat-field types are available. As a result, AWE offers three different combination methods:

1. The MasterflatFrame is constructed by extracting high spatial frequency components from The DomeF latFrame and low spatial frequencies from the TwilightFlatFrame, multiplied to give the master flat

2. The MasterFlatFrame is a direct copy of the DomeF latFrame

3. The MasterFlatFrame is a direct copy of the TwilightFlatFrame

In all cases a NightSkyFlatFrame can be provided which is multiplied with this master flat-field as an improvement on it as mentioned above.

In certain situations, it may be advantageous to split the DomeF latFrame and TwilightFlatFrame contributions out of the process. The machinery of $A W E$ allows this to be accomplished in a straight-forward manner. The advantages to this would be in isolating either large-scale (low spatial frequencies) or small-scale (high spatial frequencies), pixel-to-pixel variations of the TwilightFlatFrame or DomeFlatFrame, respectively. This concept will be explored further in Section 2.8.4.

To give a more detailed description, low spatial frequencies are extracted from the master dome and master twilight flats by the process indicated below. The high spatial frequencies of the dome flat are obtained by dividing the dome flat by its low spatial frequency components. The low spatial frequencies of 
the twilight flat are then multiplied by the high spatial frequencies of the dome flat.

Low spatial frequencies are extracted as follows:

- All bad pixels in input images are replaced by the median value of the pixels in a box around the bad pixel

- To reduce problems with Fourier filtering near image edges the size of the image is increased by mirroring the edges and corners

- A two-dimensional array is created containing the equivalent of a circular Gaussian convolution function in Fourier space (taking into account the quadrant shift introduced by the Fourier transform)

- The Fourier transform of the image is multiplied by the Gaussian filter

- The image is transformed back, and the mirrored regions removed

- The resulting image is normalized, excluding bad pixel values

\subsubsection{FringeFrame}

Fringing requires a different approach to background subtraction. Fringing in a solid state detector chip is due to interference of incident photons with photons reflected in the detector chip substrate. The photons causing the strongest fringes are those of several skylines, mostly apparent at the long wavelengths, that can vary with filter. Normally, after flatfielding, the background can be expected to be flat over the entire image, and a median of the image, excluding $5 \sigma$ outliers, would in principle be sufficient to subtract the background.

In images that suffer from fringing we have to deal with a background that is variable on small $\left(\ll 1^{\prime}\right)$ scales within the image, and can not be distinguished from sources. The image itself can, therefore, not be used to determine the background. However, the information of several images can be combined to determine a background. This average should include enough observations to properly exclude contamination from sources.

A suitable strategy to construct a fringed background image, usable for subtraction, thereby removing the fringe pattern, remains to be determined. If the fringe pattern is stable over the night, a decomposition of the nightsky flat in an additive and multiplicative term is feasible. The assumption that the high-frequency spatial component in the night-sky flat are fringes, while the lowest frequency components represent gain variations has been used with reasonable success.

The procedure to create a FringeFrame starts with a minimum of three non-cospatial science images of reasonably long exposure time (e.g., >30 s) within a given night in a given band to achieve optimal results. Images are overscan-corrected, trimmed, debiased, flat-fielded and normalized, then stacked and a median along the $Z$-axis is calculated. This median is intended to remove any non-systematic effects (objects, cosmic rays, satellite tracks, etc.). The median image is then normalized to the mean taking into account hot and cold pixels. The value of 1.0 is subtracted from the normalized fringe map to 
obtain an average value of zero. Bad pixels are assigned a value of zero by multiplying by the combined hot and cold pixel maps.

During a night the brightness of the emission lines will change, especially near evening and morning twilight. The result of this is that the amplitude of the observed fringes will change. Therefore, fringe maps should be scaled to fit the amplitude of the fringes in each science frame. This is calculated from the standard deviation in a science image, which is derived from all non-bad pixels that have values within a given threshold from the median background level. It is assumed that this standard deviation depends on the amplitude of the fringes.

\subsection{AstrometricParameters}

Astrometric calibration is a vital, integral part of any astronomical data reduction and analysis system. AWE performs two kinds of astrometric calibration of pixel data. Their results are termed local astrometry and global astrometry. The goal of the global astrometry is to improve on the local astrometry. Unlike all the previous calibrations, the resulting AstrometricParameters objects are each linked to a single processed science observation (a single detector chip of one exposure), as it is that observation that provides the source positions to be calibrated via the astrometric solution.

The local astrometric solution (see Section 2.7.1) is derived on the basis of a single detector chip's information. It is obtained by minimizing the differences between the RA and DEC positions of sources in a single detector chip and their positions listed in a catalog of astrometric standards. The global astrometric solution (see Section 2.7.2) can be obtained if one has dithered (nearly cospatial and cotemporal) observations and local astrometric solutions for each detector chip. It then additionally minimizes the positional differences of sources appearing on more than one detector chip. This results in a higher accuracy of the astrometric calibration. The use of global astrometry improves the image quality of a coaddition of dithered observations compared to local astrometry.

In AWE, astrometric solutions are solved by running LDAC (Leiden Data Analysis Center ${ }^{11}$ ) $\mathrm{C}$ programs on catalogs extracted from reduced pixel data. The $\mathrm{C}$ programs are wrapped in Python to allow interaction with the objectoriented database model employed by AWE. In local astrometry, all the steps in the astrometric solution (pre-astrometric correction, association, formal solution, etc.) are handled by the LDAC programs. In global astrometry, all the steps are also handled by LDAC except for the initial cross-correlation (called association) of sources which is handled by the AWE database (via

\footnotetext{
${ }^{11} \mathrm{ftp} / / / \mathrm{ftp}$. strw.leidenuniv.nl/pub/ldac/software/pipeline.pdf
} 
advanced queries). This offers a performance advantage because the data to be associated already resides in the database to be used in any combination as needed.

\subsubsection{Local astrometry}

Local astrometry in AWE starts with a ReducedScienceFrame that has some basic astrometry, directly from the telescope or updated sometime prior ingestion. In a parallel environment, the ReducedScienceFrame is run through the AstrometricParametersTask, a Python convenience recipe interacting with the database, whereby various $C$ programs wrapped in Python solve for the astrometry on the catalog level. SExtractor is run to extract the initial catalog. After this, LDAC tools perform all subsequent operations: preastrometric fitting to solve for large (approximately arcminute level) offsets, scaling, and rotations using the any all-sky catalog for reference (e.g., USNO, 2MASS, etc.). This pre-astrometry is then applied to the catalog and it is formally associated with the reference catalog with offsets that are now on the order of arcseconds. During the process, only the most stellar-like and best quality objects, as determined by SExtractor flags (for saturation, incomplete objects on the edge of a detector chip, blended objects, etc.) are retained. The catalog is then run through the LDAC. astrom program where the final astrometry is determined (least-squares fit to a 2-degree polynomial) and residuals catalog created. The last step is converting the distortion correction to world coordinates prior to storing the solution parameters in the database and the residuals catalog on the dataserver. These final residuals are now on the level of accuracy of the reference catalog used.

The residuals catalog output from the LDAC.astrom program contains residuals of the form DRA $=\mathrm{RA}_{\text {ref }}-\mathrm{RA}_{\mathrm{ldac}}$ and $\mathrm{DDEC}=\mathrm{DEC}_{\mathrm{ref}}-\mathrm{DEC}_{\mathrm{ldac}}$, where $\mathrm{RA}_{\text {Idac }}$ and $\mathrm{DEC}_{\text {ldac }}$ are the coordinates of the extracted sources, corrected for all distortions by the LDAC programs, and $\mathrm{RA}_{\text {ref }}$ and $\mathrm{DEC}_{\text {ref }}$ are the coordinates of the reference sources from the reference catalog used. The residual plots created by the AstrometricParameters inspect method plot information directly from this residuals catalog and show what is to be the expected precision of the correction when the ReducedScienceFrame is regridded into a RegriddedFrame. After the local astrometric solution is created, the information can be applied to create a RegriddedFrame (see Section 3.5) and eventually a CoaddedRegriddedFrame (see Section 3.6).

\subsubsection{Global astrometry}

The most important concept in the global solution in AWE is that it is local. It is local in the sense that it uses the extra information of a set of dithered observations that are closely matched both temporally and spatially (e.g., exposures taken within one to $2 \mathrm{~h}$ with more than $90 \%$ of each detector 
chip participating in the overlap region, respectively). ${ }^{12}$ The extra information characteristic of a closely matched dither consists of the smooth variations in time of the optical system distortions and the large amount of overlap of the detector area. Combining the distortion information with the overlap information allows the global solution to attain the higher precision needed for proper coaddition of the source frames. This local-global astrometry is the only method of global astrometry certified in AWE.

The process of global-global astrometry is quite different. It involves combining those dithers from widely different observation times, using independent derivations of the optical system distortions, but combining all overlap information available from overlapping dithers. It allows for the discontinuity among dithers that the local-global process cannot. This type of global astrometry is not present in AWE at this time.

Global astrometry in AWE starts with the GAstromSourceListTask, a Python convenience recipe interacting with the database that creates a special Sourcelist (see Section 3.7) from the source ReducedScienceFrame using the AstrometricParameters information created by the local solution. This is done in a parallel environment and only if the Sourcelists don't already exist. Next, the GAstromTask recipe is run in a serial environment as only a single thread is needed. It associates the source position information from the associated Sourcelist, residing solely in the database, using an Associatelist object (see Section 3.8). This step replaces the LDAC.associate stage in the local solution. After the association, LDAC astrom is run on the associated data using a least-squares fit to a 3degree polynomial (as opposed to a 2-degree polynomial in the local solution), and like the local solution, a residuals catalog is created.

The residuals catalog output from the LDAC . astrom program in this case, contains two sets of residuals, one identical to that of the local solution with respect to the reference catalog used (see Section 2.7.1), and the other with respect to the overlapping extracted sources. The latter residuals are of the form DRA $=\mathrm{RA}_{2}-\mathrm{RA}_{1}$ and $\mathrm{DDEC}=\mathrm{DEC}_{2}-\mathrm{DEC}_{1}$, where $\mathrm{RA}_{1}$ and $\mathrm{DEC}_{1}$ are the coordinates of the extracted sources from a given frame and $\mathrm{RA}_{2}$ and $\mathrm{DEC}_{2}$ are the coordinates of the extracted sources from another pointing, same or different detector chip, that overlaps the first, both corrected for all distortions by the LDAC. astrom program. The residual plots created by the GAstrometric inspect method plots both sets of residuals directly from this residuals catalog, both by individual detector chip and for all detector chips

\footnotetext{
${ }^{12}$ Global astrometry in AWE is based on the concept of fixed focal-plane geometry. This means that any difference in the apparent focal plane from pointing to pointing is assumed to change in a linear fashion only, with higher order distortions remaining constant (i.e., only relative translations of the entire focal plane in RA and Dec are corrected for). This asumption of fixed focal-plane geometry adds information to the system, benefiting the astrometric solution. Generally, only sets of exposures taken temporally close and spatially close will match this criteria. These two conditions minimize differences in telescope flexure caused by different altitude and azimuth locations, and maximize the number of objects common to all exposures.
} 
combined, and shows what is to be the expected precision of the global solution used to combine a set of RegriddedFrames into a CoaddedRegriddedFrame.

After the global astrometric solution is created, the information is used to create a new AstrometricParameters instance for each ReducedScienceFrame that went into the solution. The parameters and statistics for the global solution are computed and stored on a per frame basis and likely will not match those values of other frames from the same solution. As with the local solution, these parameters can be applied to create RegriddedFrames (see Section 3.5) and eventually a CoaddedRegriddedFrame (see Section 3.6), but with much greater precision than with the local solution only (see Fig. 7 for an example using WFI data).

\subsection{PhotometricParameters}

The photometric pipeline in AWE is aimed at calibrating large imaging surveys taken with multi-detector chip wide-field imagers during many nights and different epochs. Instrumental characteristics specific for wide-field imagers need to be accounted for in a survey photometric pipeline. For example:

- Detector chip-to-detector chip variations. Each detector chip has its own small and large-scale variations in pixel gain and can have a different median gain. There can also be detector chip-to-detector chip variations in the non-linearity behavior of count rates or color terms of the photometric calibration.

- Illumination variation. Several wide-field imagers are known to have illumination variations (e.g., MEGACAM at CFHT, WFI at ESO/MPG 2.2.m). The gain variation over individual detector chips is characterized by flatfields under the assumption of an ideal flat illumination over the field of view. In practice this ideal flat illumination can be affected by stray and/or scattered light (sky concentration) yielding variations of up to a few tenths of a magnitude in amplitude.

- Shutter timing. The large FoV requires carefully designed shutter mechanisms. Shutter timing variations might result in position dependent exposure times.

Performing a survey with such an instrument poses several challenges for the photometric calibration. Long term, short-term, night-to-night or even intranight variations need to be monitored to create a homogeneous photometric calibration across the whole survey area and survey time. It might be the case that the very precise photometric calibration is dependent on instrumental variations not captured by a single or handful standard star observations per night, e.g., telescope altitude and azimuth. To detect and quantify all such effects it is important to explore trends in photometric results as a function of many parameters. To obtain the maximum photometric accuracy it is required to have observations of photometric standards that densely cover the full FoV. 
Fig. 7 An example of improvement from the local to the global solution. Both panels show astrometric residuals, in arcsec,

$\triangle R A=R A_{1}-R A_{2}$ and $\Delta D e c=D e c_{1}-D e c_{2}$, where $R A_{1}$ and $D E C_{1}$ are the source positions from any one frame, and $R A_{2}$ and $D E C_{2}$ are the source positions of all matching sources in one of the other frames, same or different detector chip, that overlaps the first. The top panel shows the overlapping source position differences from 32 frames of a 4-point WFI dither regridded using the local solution (limits scaled to match lower panel), the bottom panel shows the same for the same frames regridded using the global solution. The improvement in precision is greater than a factor two
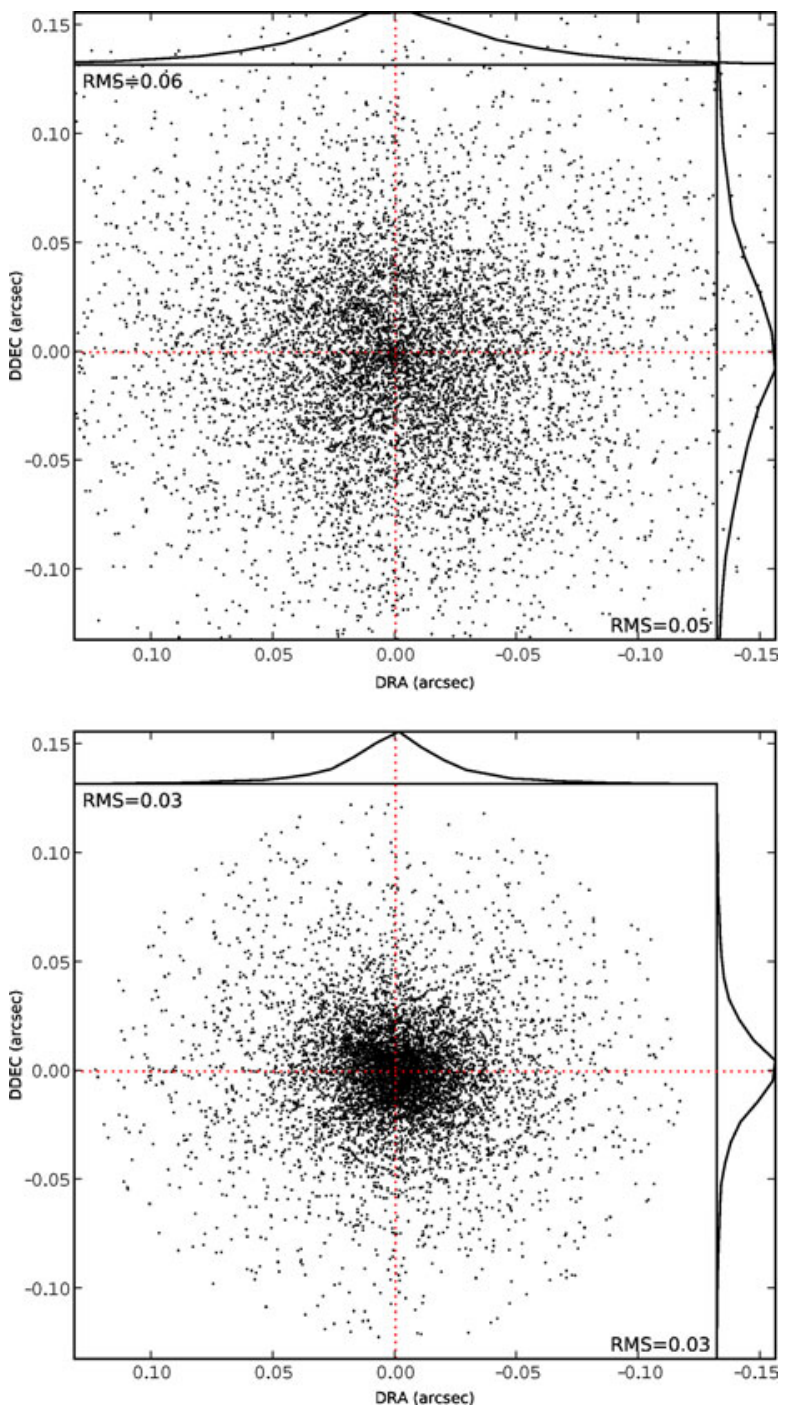

The goal of AWE photometric calibration is to establish the photometric system resulting from the signal progressing through Earth's atmosphere, telescope, filter, wide-field camera and each detector chip resulting in a digital read-out. The photometric system is characterized in AWE in terms of a multiplication of gains:

$$
\begin{aligned}
I_{\text {obs }}= & g_{\text {ff }}(t, N, X,(x, y)) \times g_{\mathrm{e}}\left(t_{0}\right) g_{\mathrm{e}}(t) g_{\text {sel.e }}(X) \times g_{\mathrm{qe}}\left(t_{0}, N, X\right) g_{\mathrm{qe}}(t, N, X) \\
& \times g_{\text {illum }}(t, N,(x, y), X) \times I_{\text {ref }},
\end{aligned}
$$

where $I_{\text {obs }}$ is the observed countrate of a standard star in digital units and $I_{\text {ref }}$ its emitted physical flux, $t$ is time and $(x, y)$ position on the detector chip. The 
gain $g_{\mathrm{ff}}(t, N, X,(x, y))$ characterizes the flat field. The gains $g_{\mathrm{e}}$ characterize the atmospheric extinction: $g_{\mathrm{e}}\left(t_{0}\right)$ is the scaling at time of the selected atmospheric extinction curve $g_{\text {sel.e }}(X)$ that is a function of filter $X$ and $g_{\mathrm{e}}(t)$ models the change at time $t$. The gains $g_{\text {qe }}$ characterize the overall instrumental quantum efficiency that includes the light losses through the optics and conversion from physical units of flux to countrates for detector chip $N$. The illumination variation is captured as a separate gain $g_{\text {illum }}$.

By determining the gains, AWE then gives for each detector chip independently the photometric calibration at any time for any pixel for each filter. The photometric calibration objects have timestamps to indicate their validity range in time (see Section 1.1). Thus AWE holds a continuous representation of the photometric system of an instrument if the calibration plan of the instrument provides the required observations. This is another example of how AWE calibrates the instrument instead of a specific data set.

The gain factors representing atmospheric extinction and instrumental quantum efficiency are solved in magnitude space. The involved physics for wide-field cameras is well-represented by the common photometric equation for astronomical imagers:

$$
\begin{aligned}
m_{\mathrm{inst}}= & -2.5 \log (\text { countrate })+Z P T-k \times A M \\
& +C_{0}-C_{1} \times\left(m_{X 2}-m_{X 3}\right)
\end{aligned}
$$

where $m_{\text {inst }}$ is the magnitude of the object in the instrumental photometric system, the countrate is in ADU/s, $\mathrm{k}$ is the atmospheric extinction coefficient, $\mathrm{AM}$ is the airmass, and $C_{0,1}$ are the terms describing the corrections to go from the standard photometric system to the instrumental photometric system. $m_{X 2}-m_{X 3}$ is the color between filter $X 2$ and $X 3$ of the standard star as listed in the catalog of the standard photometric system.

\subsubsection{Atmospheric extinction}

The atmospheric extinction in magnitude space is assumed to be a linear function of airmass AM (i.e., $k \times A M$ in (2) which is a representation of $g_{\mathrm{e}}$ in $(1)\left(g_{\mathrm{e}} \sim 10^{-(k \times A M) / 2.5}\right)$. The task is to establish the atmospheric extinction coefficient $k$. The airmass is taken from the observational metadata.

In AWE, the correction for the atmosphere in the photometric calibration can be derived in four ways.

1. Using a pre-defined atmospheric extinction coefficient. The coefficient is multiplied by the airmass (see (2)). These are stored in the AWE database for each combination of instrument and filter object of the class AtmosphericExtinctionCoefficient. Users can insert their own atmospheric extinction coefficients in AWE.

2. Using a pre-defined atmospheric extinction coefficient plus a shift. It is using the coefficient just described plus a shift given by a report represented by the class PhotometricExtinctionReport. This kind of 
atmospheric correction on the photometry is represented by the class AtmosphericExtinctionCurve.

3. Using standard star field observations. This kind of correction is represented by the class AtmosphericExtinction. There are two sub options here:

(a) Using a single standard star field observation and a given zeropoint. Equation (2) is the used to determine an atmospheric extinction coefficient. This type of atmospheric correction is represented by the AtmosphericExtinctionZeropoint class.

(b) Using two observations of standard star fields at different airmass. By equalling the zeropoint in (2) one can solve for the atmospheric extinction coefficient. This correction type is represented by the class AtmosphericExtinctionframes.

\subsubsection{Color terms}

Differences in the effective throughput per wavelength of the photometric system of the standard system and the instrumental system can be caused, for example, by differences in filter transmission curves or in quantum efficiencies of the detector chips. In AWE, it is assumed that these differences can be captured by a linear function of the standard star color in the standard photometric system:

$$
m_{\mathrm{ref}, \mathrm{i}, \mathrm{inst}}=m_{\mathrm{ref}, \mathrm{i}, \mathrm{std}}+C_{0}-C_{1} \times\left(m_{i, X 2}-m_{i, X 3}\right),
$$

where $m_{\text {ref,i,inst }}$ is the magnitude of the standard star $i$ in the instrumental photometric system and $m_{\text {ref,i,std }}$ in the standard photometric system. For each combination of instrument and filter the two coefficients are pre-determined and stored in AWE. The PhotTransformation class represents the color transformation, and objects of this class contain the coefficients. The magnitudes of the standard star in filters $X 2, X 3$ is taken from the standard star catalog (a PhotRefCatalog object) stored in AWE.

\subsubsection{Zeropoints}

The flux counts and astrometry of stars in a photometric standard field are measured using SExtractor. The resulting catalog is associated (using the prephotom package in LDAC) with known standard stars listed in a reference catalog. Now a "raw" instrumental magnitude $\left(m_{\text {raw,i,inst }}\right)$ and zeropoint $Z P T_{\text {raw,i,inst }}$ are computed for each observed standard star $i$ :

$$
\begin{array}{r}
m_{\text {raw }, \mathrm{i}, \text { inst }}=-2.5 \log \text { countrate } \\
Z P T_{\text {raw }, \text { i,inst }}=m_{\text {ref,i,inst }}-m_{\text {raw }, \mathrm{i}, \text { inst }}
\end{array}
$$

A clipping is applied on the set of raw zeropoints:

$$
\left|Z P T_{\text {raw }, \text { i,inst }}-\operatorname{median}\left(Z P T_{\text {inst,i,raw }}\right)\right|<M A X_{-} M A G_{-} D I F F,
$$


with $M A X \_M A G \_D I F F$ set by the user. The result is stored in a photometric source catalog represented by the class PhotsrcCatalog.

If at least a required minimum number of standard stars identified in the observation remain (the MIN_NMBR_OF_STARS parameter), the final zeropoint is computed. A sigma clipping with a threshold factor SIGCLIP_LEVEL set by the user is applied once to the raw zeropoints. The variance weighted mean and its uncertainty are computed from the remaining raw zeropoints. This mean is then corrected for the atmospheric extinction yielding the zeropoint $Z P T$. The $Z P T$ is stored in a PhotometricParameters object. Formal errors are propagated from count measurements through the computation of zeropoint and atmospheric extinction.

AWE contains a photometric reference catalog that contains the magnitudes of standard stars in Johnson-Cousins system (from Landolt and Stetson), and the Sloan system in 22 SA fields. By default, all entries are used from in the standard star catalog, but one can limit this to subsets. It is also possible to use a custom photometric reference catalog.

\subsubsection{IlluminationCorrection}

The photometric calibration accounts for gain variations under the assumption of an ideal flat illumination over the field of view. In practice this ideal flat illumination can be affected by stray light (sky concentration) and a correction for this effect has to be made.

It is assumed that the effect of the illumination variations is larger than detector chip-to-detector chip systematic variations. It has been verified that this holds for the MEGACAM and WFI instruments. The starting point is all detectors of a mosaic of a standard star field observation that is detrended up to the flat field level in a given filter. The raw zeropoint (see Section 2.8.3) is determined for each standard star. The residual between these zeropoints and their median value over all detector chips in the mosaic is a measure of the illumination variation. The residual distribution is assumed to be well-fitted with a two-dimensional second order polynomial (as is verified for MEGACAM and WFI) using a chi-square minimization. An illumination variation frame is created from the polynomial fit for each detector chip. Each standard star field frame is divided by this IlluminationCorrectionFrame and a new zeropoint determination is performed per detector chip. This last step corrects for any remaining detector chip-to-detector chip variations.

The resulting illumination correction is applied to ReducedScienceFrames in the following manner: the background is removed from the science frames and the remaining pixels associated with sources (both calculated by SExtractor) are multiplied by the IlluminationCorrectionframe. The background is added back and the zeropoints from the standard star field with illumination correction are applied.

In wide-field instruments (e.g., OmegaCAM), the illumination variation pattern across the large detector block can vary with time, telescope position, etc. In these cases, an IlluminationCorrectionFrame may fail to 
properly characterize the illumination variation and require a different approach. One such approach involves compensating for only the pixel-to-pixel variations in the flat-fielding as alluded to in Section 2.6.4. A MasterFlatFrame constructed from only the high spatial frequencies of a DomeFlatFrame can be used to eliminate the pixel-to-pixel sensitivity variations without adding any illumination variation from the low spatial frequency (large scale) contributions. Any remaining illumination variation above the background, if it exists, can then be corrected for appropriately, either as described above or via robust sky subtraction techniques (e.g., with SExtractor).

\section{Image pipeline: combining the pixels}

As mentioned earlier, one advantage of the AWE is its parallel processing capability. Much of the processing is done in a parallel environment, one detector chip per CPU node. There are two places in the image pipeline, however, where the information of individual detector chips must be combined: the astrometric solution may be derived for all detector chips simultaneously (global astrometry), and science images may be coadded into larger mosaics and/or deeper images. See Fig. 8 for an overview.

Many ProcessTarget's have configurable processing parameters to control how they are processed. Table 3 gives an overview of these process_params for the image pipeline.

\subsection{ReducedScienceFrame}

The most basic outcome of the image pipeline is the ReducedScienceFrame. Conventional de-trending steps are performed when making this frame:

1. Overscan correction and trimming

2. Subtraction of the Biasframe

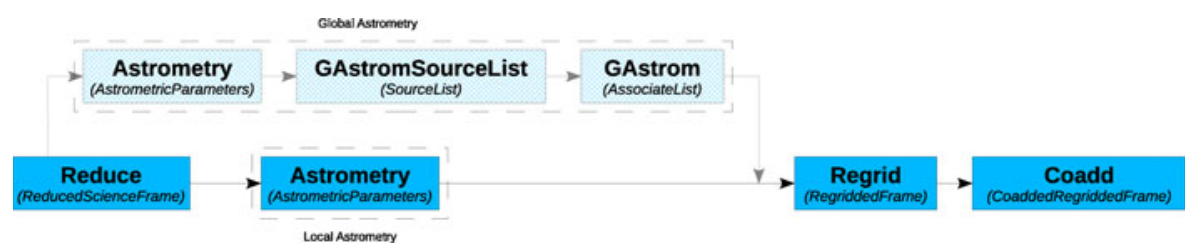

Fig. 8 Schematic flow of the image pipeline following the coloring in Fig. 1. The recipes, also called Tasks, used to produce various ProcessTargets are indicated in each box (with their data product in parentheses) and described in the various sections. The arrows connecting them indicate the direction of processing. Note that the global (multi-chip) astrometry branch is optional and supplementary to the local (single-chip) astrometry. Also note, that while AssociateList is the formal data product of GAstrom, new AstrometricParameters objects are created in the process as well 
Table 3 Processing parameters and their default values

\begin{tabular}{|c|c|c|c|}
\hline Class & process_param & Value & Units \\
\hline \multirow[t]{4}{*}{ ReducedScienceFrame } & overscan_correction & 6 & \\
\hline & fringe_threshold_low & 1.5 & \\
\hline & fringe_threshold_high & 5.0 & \\
\hline & image_threshold & 5.0 & \\
\hline \multirow[t]{2}{*}{ SaturatedPixelMap } & threshold_low & 50.0 & $\mathrm{ADU}$ \\
\hline & threshold_high & 50000.0 & $\mathrm{ADU}$ \\
\hline \multirow[t]{2}{*}{ Satellitemap } & detection_threshold & 5.0 & \\
\hline & hough_threshold & 1000.0 & \\
\hline RegriddedFrame & background_subtraction_type & 0 & \\
\hline SourceList & htm_depth & 25 & \\
\hline \multirow[t]{3}{*}{ AssociateList } & search_distance & 5.0 & $\operatorname{arcsec}$ \\
\hline & single_out_closest_pairs & 1 & \\
\hline & sextractor_flag_mask & 0 & \\
\hline
\end{tabular}

These values are representative of the typical value for any instrument. Some instruments may have values that different from these based on experience with that instrument. See the document page linked from the class name or appropriate links on http://doc.astro-wise.org/astro.main.html for more details

3. Division by the MasterflatFrame

4. Scaling and subtraction of a FringeFrame if indicated

5. Multiplication by an IlluminationCorrectionFrame if indicated

6. Creation of the individual weight image

7. Computation of the image statistics

Please note that:

- The overscan correction can be a null correction (i.e., no modification of the pixel values)

- The illumination correction step (i.e., application of a photometric flat field) has had a SExtractor-created background removed and then reapplied after the multiplication, and the correction only occurs when requested and if a suitable IlluminationCorrectionframe exists

\subsection{WeightFrame}

In addition to the effects of hot and cold pixels, individual images may be contaminated by saturated pixels, cosmic ray events, and satellite tracks. For purposes of subsequent analysis and image combination, affected pixels unique to each image need to be assigned a weight of zero in that image's weight map.

Since the variance is inversely proportional to the Gain, which is proportional to the flatfield, the weight is given by:

$$
W_{i j}=G_{i j} P_{\text {hot }} P_{\text {cold }} P_{\text {saturated }} P_{\text {cosmic }} P_{\text {satellite }},
$$

where $W_{i j}$ is the weight of a given pixel, $G_{i j}$ is the gain of a given pixel (taken from the flat field), and the rest of the members are binary maps where good pixels have a value of 1 and bad pixels have a value of 0 . These maps are, 
respectively, a HotPixelMap, a ColdPixelMap, a SaturatedPixelMap, a CosmicMap, and a SatelliteMap, the last three being calculated directly from the ReducedScienceFrame after detrending.

\subsubsection{SaturatedPixelMap}

Saturated pixels are pixels whose counts exceed a certain threshold. In addition, saturation of a pixel may lead to dead neighbouring pixels, whose counts lie below a lower threshold. These upper and lower thresholds are defined and stored in the object.

\subsubsection{CosmicMap}

Two programs may be used to detect cosmic ray events:

1. SExtractor can be run with a special filter that is only sensitive to cosmicray-like signal. This requires a 'retina' filter, which is a neural network that uses the relative signal in neighboring pixels to decide if a pixel is a cosmic. A retina filter, called 'cosmic.ret' is provided. Run SExtractor with FILTER_NAME=COSmic.ret, to run SExtractor in comic ray detection mode. This results in a so-called segmentation map, recording the pixels affected by cosmic ray events. This segmentation can be used to assign a weight of zero to these pixels.

2. CosmicFITS is designed as a stand-alone program to detect cosmic ray events.

In the AWE, the SExtractor method is the preferred cosmic ray event detection method.

\subsubsection{Satellitemap}

Linear features can be detected using a Hough transform algorithm, which is used to find satellite tracks. See Duda and Hart [2], Hough [3] for more information about the Hough transform.

A point $(x, y)$ defines a curve in Hough space $(\rho, \theta)$, where:

$$
\rho=x \cos \theta+y \sin \theta,
$$

corresponding to lines with slopes $0<\theta<\pi$, passing at a distance $\rho$ from the origin. This means that different points lying on a straight line in image space, will correspond to a single point $(\rho, \theta)$ in Hough space.

The algorithm then creates a Hough image from an input image, by adding a Hough curve for each input pixel which lies above a given threshold. This Hough image (effectively a histogram of pixels corresponding to possible lines) is clipped, and transformed back into a pixelmap, masking lines with too many contributing pixels. 


\subsection{AstrometricParameters}

The parameters from the astrometric solution are used during the regridding process and their creation has already been discussed in Section 2.7.

\subsection{PhotometricParameters}

The parameters from the photometric solution are used during the coaddition process and their creation has already been discussed in Section 2.8.

\subsection{RegriddedFrame}

Regridding and co-adding are done using the $S W \operatorname{arp}^{13}$ program. Before images are co-added, they are resampled to a predefined pixel grid (see Appendix). By co-adding onto a simple coordinate system, characterized by the projection (Tangential, Conic-Equal-Area), reference coordinates, reference pixel, and pixel scale, the distortions recorded by the astrometric solution are removed from the images. To this end a set of projection centers is defined, at $1^{\circ}$ separation and pixel scale of 0.2 arcsec. A ReducedScienceFrame resampled to this grid is called a RegriddedFrame. The background of the image can be calculated and subtracted at this time, if desired.

\subsection{CoaddedRegriddedFrame}

After the RegriddedFrames are made, it is only a matter of applying the photometry of each frame and stacking the result. This process creates a CoaddedRegriddedFrame.

One point of great importance in considering the coadded data is its pixel units. The units are fluxes relative to the flux corresponding to magnitude $=0$. In other words, the magnitude $m$ corresponding to a pixel value $f_{0}$ is:

$$
m=-2.5 \log _{10} f_{0}
$$

The value $f_{\text {out }}$ of a pixel in the CoaddedRegriddedFrame is computed from all overlapping pixels $i$ in the input RegriddedFrames according to this formula:

$$
f_{\text {out }}=\Sigma_{i}\left(w_{i} * F L X S C A L E_{i} * f_{i}\right) / \Sigma_{i}\left(w_{i}\right),
$$

where $f_{i}$ is the pixel value in the RegriddedFrame, FLXSCALE $E_{i}$ is calculated from the zeropoint, and $w_{i}=$ weight $_{i} / F L X S C A L E_{i}^{2}$ where weight $_{i}$ is the value of the pixel in the input weight image. A WeightFrame is created as well. The value $w_{\text {out }}$ of the pixel in the weight frame for the coadd is:

$$
w_{\text {out }}=\Sigma_{i}\left(w_{i}\right)
$$

\footnotetext{
${ }^{13} \mathrm{http}: / /$ astromatic.iap.fr/software/swarp/
} 


\subsection{Sourcelist}

In AWE, source information from processed frames can be stored in the database in the form of Sourcelists. These are simply a transcription of a SExtractor-derived catalog values (position, ellipticity, brightness, etc.) into the database. Normally, the catalog was derived from a processed frame existing in the system, but this is not a requirement. Arbitrary SExtractor catalogs meeting a minimum content criteria can be ingested as well. This is how large survey results and reference catalogs are brought into the system.

These Sourcelists can be used for a variety of purposes such as astrometric and photometric correction, but are normally an end product of the image pipeline storing key quantities about the sources in question for further analysis. Multiple Sourcelists can be combined into an Associatelist, and later into another Sourcelist via the CombinedList machinery.

\subsection{Associatelist}

Multiple Source Lists can be spatially combined (VIA RA and DEC values) and stored in the database via the Associatelist class. The association is done in the following way:

1. The area of overlap of the two Sourcelists is calculated. If there is no overlap no associating will be done.

2. The sources in one Sourcelist are paired with sources in the other if they are within a certain association radius. Default radius is $5 \%$. The pairs get an unique associate ID (AID) and are stored in the AssociateList. A filter is used to select only the closest pairs.

3. Finally the sources which are not paired with sources in the other list and are inside the overlapping area of the two Sourcelist are stored in the Associatelist as singles. They too get an unique AID.

Very important is the type of association being done. One of three types: chain, master or matched, will be done. In a chain association, all subsequent Sourcelists are matched to the previous Sourcelist to find pairs, in a master association, they are always matched with the first SourceList, and in a matched association, all SourceLists are matched with all other SourceLists.

\section{Summary}

The development and implementation of the Astro-WISE optical pipeline has been described. This pipeline uses the Astro-WISE Environment: an information system designed to integrate hardware, software and human resources, data processing, and quality control in a coherent system that provides an unparalleled environment for processing astronomical data at any level, be it 
an individual user or a large survey team spread over many institutes and/or countries.

The Astro-WISE Environment is built around an Object-Oriented Programming (OOP) model using Python where each data product is represented by the instantiation of a particular type of object. The processability and quality of these data objects (ProcessTargets) is moderated by built-in attributes and methods that know, for each individual type of object or OOP class, how to process or qualify itself. All progenitor and derived data products are transparently linked via the database, providing an uninterrupted path between completely raw and fully processed data.

This data lineage and provenance allows for a type of processing whereby the pipeline used for a given set of data is created on-the-fly for that particular set of data, where the Unix make metaphor is employed to chain backward though the data, processing only what needs to be processed (target processing). This allows unparalleled efficiency and data transparency for reprocessing the data when necessary, as the raw data is always available when newer techniques become available.

Calibration of data follows the usual routes, but has been optimized for processing of OmegaCAM calibration data meant for detrending survey data. In this process, data is processed and reprocessed as more and more knowledge of the instrument system (from the optics through detector chain) is acquired. This effectively calibrates the instrument, leaving the data simply to be processed without the need of users find or qualify their own calibrations. Various attributes of calibration objects (validity, quality, valid time ranges) transparently determine which calibrations are best to be used for any data. Processing parameters are set and can be reset as desired. These parameters are retained as part of the calibration object and guarantee that a given object can be reprocessed to obtain the same result or be tweaked to improve the result. The processing of science data is governed by the same validity, quality, valid time range, and processing parameter mechanism that is used for calibration data.

The calibration pipeline starts with a ReadNoise object created from RawBiasframes that is used to determine a clipping limit for BiasFrame creation. A GainLinearity object can be processed from a special set of RawDomeFlatFrames taken for the purpose. From this result, both the gain (in $e^{-} / \mathrm{ADU}$ ) and the detector linearity can be determined. A master BiasFrame is created from a set of RawBiasframes to remove 2-dimensional additive structure in detectors. The DarkCurrent is measured for quality control of the detectors, but is not applied to the pixels. Bad pixels in a given detector can be found from the BiasFrame and a flat field image. These are termed HotPixelMap and ColdPixelMap, respectively.

Flat field creation in Astro-WISE can be very simple or very complex. On the simple side, a single set of RawDomeF latFrames or RawTwilightFlatFrames can be combined with outlier rejection and normalized to the median. On the complex side, high spatial frequencies can be taken from the DomeFlatFrame and the low spatial frequencies from the TwilightFlatFrame. 
In addition, a NightSkyFlatFrame can be added to improve this result. For an additional refinement to the flat field correction for redder filters, a FringeFrame can be created.

Astrometric calibration starts with extraction of sources from individual ReducedScienceframes. The source positions are matched to those in an astrometric reference catalog (e.g., USNO-A2.0) and all the positional differences minimized with the LDAC programs. This local solution can then be further refined by adding overlap information from a dither to form a global astrometric solution. Astrometric solutions are always stored for each ReducedScienceframe individually. Photometric calibration also starts with source extraction (as a PhotSrcCatalog) and positional association. Then, the magnitudes of the associated sources are compared to those in a photometric reference catalog (e.g., Landolt) and the mean of the Kappasigma-clipped values results in a zeropoint for a given detector for the night in question. The extinction can be derived from multiple such measurements, the results of both being stored in a PhotometricParameters object. As an optional refinement to the photometric zeropoint, a photometric super flat can be constructed by fitting magnitude differences as a function of radius across the whole detector block. The result of this is stored in an IlluminationCorrectionFrame object.

The image pipeline takes all the calibrations from BiasFrame through MasterFlatFrame to transform a RawScienceFrame into a ReducedScienceFrame. This includes trimming the image after applying the overscan correction, subtracting the BiasFrame, dividing by the MasterF latFrame, and applying the FringeFrame and IlluminationCorrectionFrame if necessary. The WeightFrame is constructed by taking the HotPixelMap and ColdPixelMap and combining them with a SaturatedPixelMap, a SatelliteMap, a CosmicMap, and optionally a IlluminationCorrectionframe. These are all applied to the MasterflatFrame to create the final WeightFrame. Next, the AstrometricParameters is applied to the ReducedScienceFrame in creating the RegriddedFrame, and the PhotometricParameters is applied to multiple RegriddedFrames to form a CoaddedRegriddedFrame. Lastly, the sources from one CoaddedRegriddedFrame can be extracted into a Sourcelist and associated with other Sourcelists to form an AssociateList object. This last is the final output of the image pipeline and can combine information from multiple filters on the same part of the sky into one data product.

Using AWE, The KIDS survey team has begun processing each week's worth of data taken at the VST (more than half a terabyte) in a single night. The part of the data that requires it (bad quality or validity) is reprocessed nightly as necessary to gain the required insight into the different aspects of the calibration process: detrending calibrations, astrometric calibrations, and photometric calibrations.

The Astro-WISE Environment is a unique multi-purpose pipeline for astronomical surveys. All required tools (ingestion, processing, quality control, and publishing) are integrated in an intuitive and transparent way. It has already 
been used to process archive WFI@2.2m, MegaCam@CFHT (CFHTLS), and VIRCam@VISTA data in pseudo-survey mode in preparation for its main task: processing KIDS, Vesuvio, OmegaWhite, and OmegaTrans survey data from the newly commissioned OmegaCAM@VST.

Acknowledgements Astro-WISE is an on-going project which started from a FP5 RTD programme funded by the EC Action "Enhancing Access to Research Infrastructures". This work is supported by FP7 specific programme "Capacities-Optimising the use and development of research infrastructures". Special thanks to Francisco Valdes for his constructive comments.

Open Access This article is distributed under the terms of the Creative Commons Attribution Noncommercial License which permits any noncommercial use, distribution, and reproduction in any medium, provided the original author(s) and source are credited.

\section{Appendix: skygrid of projection centers}

Table 4 describe a grid on the sky for projection and co-addition purposes in a condensed format. It contains 95 strips as function of decreasing declination $\left(0^{\circ} \geq \delta \geq-90^{\circ}\right)$. For each strip the size in degrees and the number of $1^{\circ} \times 1^{\circ}$

Table 4 Strips 1-95

\begin{tabular}{|c|c|c|c|c|}
\hline Strip & $-\delta[0]$ & Size [o] & Fields/strip & Overlap [\%] \\
\hline 1 & 0.00 & 360.00 & 378 & 5.0 \\
\hline 2 & 0.96 & 359.95 & 378 & 5.0 \\
\hline 3 & 1.91 & 359.80 & 378 & 5.1 \\
\hline 4 & 2.87 & 359.55 & 378 & 5.1 \\
\hline 5 & 3.83 & 359.20 & 377 & 5.0 \\
\hline 6 & 4.79 & 358.74 & 376 & 4.8 \\
\hline 7 & 5.74 & 358.19 & 375 & 4.7 \\
\hline 8 & 6.70 & 357.54 & 374 & 4.6 \\
\hline 9 & 7.66 & 356.79 & 373 & 4.5 \\
\hline 10 & 8.62 & 355.94 & 372 & 4.5 \\
\hline 11 & 9.57 & 354.99 & 371 & 4.5 \\
\hline 12 & 10.53 & 353.94 & 370 & 4.5 \\
\hline 13 & 11.49 & 352.79 & 369 & 4.6 \\
\hline 14 & 12.45 & 351.54 & 368 & 4.7 \\
\hline 15 & 13.40 & 350.19 & 367 & 4.8 \\
\hline 16 & 14.36 & 348.75 & 366 & 4.9 \\
\hline 17 & 15.32 & 347.21 & 365 & 5.1 \\
\hline 18 & 16.28 & 345.57 & 363 & 5.0 \\
\hline 19 & 17.23 & 343.84 & 361 & 5.0 \\
\hline 20 & 18.19 & 342.01 & 359 & 5.0 \\
\hline 21 & 19.15 & 340.08 & 357 & 5.0 \\
\hline 22 & 20.11 & 338.06 & 355 & 5.0 \\
\hline 23 & 21.06 & 335.95 & 353 & 5.1 \\
\hline 24 & 22.02 & 333.74 & 350 & 4.9 \\
\hline 25 & 22.98 & 331.43 & 347 & 4.7 \\
\hline 26 & 23.94 & 329.04 & 344 & 4.5 \\
\hline 27 & 24.89 & 326.55 & 341 & 4.4 \\
\hline 28 & 25.85 & 323.97 & 338 & 4.3 \\
\hline 29 & 26.81 & 321.31 & 335 & 4.3 \\
\hline 30 & 27.77 & 318.55 & 332 & 4.2 \\
\hline
\end{tabular}


Table 4 (continued)

\begin{tabular}{|c|c|c|c|c|}
\hline Strip & $-\delta[\circ]$ & Size [o] & Fields/strip & Overlap [\%] \\
\hline 31 & 28.72 & 315.70 & 329 & 4.2 \\
\hline 32 & 29.68 & 312.77 & 326 & 4.2 \\
\hline 33 & 30.64 & 309.74 & 323 & 4.3 \\
\hline 34 & 31.60 & 306.64 & 320 & 4.4 \\
\hline 35 & 32.55 & 303.44 & 317 & 4.5 \\
\hline 36 & 33.51 & 300.16 & 314 & 4.6 \\
\hline 37 & 34.47 & 296.80 & 311 & 4.8 \\
\hline 38 & 35.43 & 293.35 & 308 & 5.0 \\
\hline 39 & 36.38 & 289.83 & 304 & 4.9 \\
\hline 40 & 37.34 & 286.22 & 300 & 4.8 \\
\hline 41 & 38.30 & 282.53 & 296 & 4.8 \\
\hline 42 & 39.26 & 278.76 & 292 & 4.7 \\
\hline 43 & 40.21 & 274.91 & 288 & 4.8 \\
\hline 44 & 41.17 & 270.99 & 284 & 4.8 \\
\hline 45 & 42.13 & 266.99 & 280 & 4.9 \\
\hline 46 & 43.09 & 262.92 & 276 & 5.0 \\
\hline 47 & 44.04 & 258.78 & 272 & 5.1 \\
\hline 48 & 45.00 & 254.56 & 267 & 4.9 \\
\hline 49 & 45.96 & 250.27 & 262 & 4.7 \\
\hline 50 & 46.91 & 245.91 & 257 & 4.5 \\
\hline 51 & 47.87 & 241.48 & 252 & 4.4 \\
\hline 52 & 48.83 & 236.99 & 247 & 4.2 \\
\hline 53 & 49.79 & 232.43 & 242 & 4.1 \\
\hline 54 & 50.74 & 227.80 & 237 & 4.0 \\
\hline 55 & 51.70 & 223.11 & 232 & 4.0 \\
\hline 56 & 52.66 & 218.36 & 227 & 4.0 \\
\hline 57 & 53.62 & 213.54 & 222 & 4.0 \\
\hline 58 & 54.57 & 208.67 & 217 & 4.0 \\
\hline 59 & 55.53 & 203.74 & 212 & 4.1 \\
\hline 60 & 56.49 & 198.75 & 207 & 4.1 \\
\hline 61 & 57.45 & 193.71 & 202 & 4.3 \\
\hline 62 & 58.40 & 188.61 & 197 & 4.4 \\
\hline 63 & 59.36 & 183.46 & 192 & 4.7 \\
\hline 64 & 60.32 & 178.26 & 187 & 4.9 \\
\hline 65 & 61.28 & 173.01 & 182 & 5.2 \\
\hline 66 & 62.23 & 167.71 & 176 & 4.9 \\
\hline 67 & 63.19 & 162.36 & 170 & 4.7 \\
\hline 68 & 64.15 & 156.97 & 164 & 4.5 \\
\hline 69 & 65.11 & 151.54 & 158 & 4.3 \\
\hline 70 & 66.06 & 146.06 & 152 & 4.1 \\
\hline 71 & 67.02 & 140.54 & 146 & 3.9 \\
\hline 72 & 67.98 & 134.98 & 140 & 3.7 \\
\hline 73 & 68.94 & 129.39 & 134 & 3.6 \\
\hline 74 & 69.89 & 123.76 & 128 & 3.4 \\
\hline 75 & 70.85 & 118.09 & 122 & 3.3 \\
\hline 76 & 71.81 & 112.39 & 116 & 3.2 \\
\hline 77 & 72.77 & 106.66 & 110 & 3.1 \\
\hline 78 & 73.72 & 100.90 & 104 & 3.1 \\
\hline 79 & 74.68 & 95.11 & 98 & 3.0 \\
\hline 80 & 75.64 & 89.30 & 92 & 3.0 \\
\hline 81 & 76.60 & 83.46 & 86 & 3.0 \\
\hline 82 & 77.55 & 77.59 & 80 & 3.1 \\
\hline 83 & 78.51 & 71.71 & 74 & 3.2 \\
\hline 84 & 79.47 & 65.80 & 68 & 3.3 \\
\hline 85 & 80.43 & 59.88 & 62 & 3.5 \\
\hline 86 & 81.38 & 53.94 & 56 & 3.8 \\
\hline
\end{tabular}


Table 4 (continued)

\begin{tabular}{llccc}
\hline Strip & $-\delta[\circ]$ & Size [o] & Fields/strip & Overlap [\%] \\
\hline 87 & 82.34 & 47.98 & 50 & 4.2 \\
88 & 83.30 & 42.01 & 44 & 4.7 \\
89 & 84.26 & 36.03 & 38 & 5.5 \\
90 & 85.21 & 30.04 & 32 & 6.5 \\
91 & 86.17 & 24.05 & 26 & 8.1 \\
92 & 87.13 & 18.04 & 19 & 5.3 \\
93 & 88.09 & 12.03 & 13 & 8.1 \\
94 & 89.04 & 6.02 & 7 & 16.4 \\
95 & 89.90 & 0.63 & 1 & - \\
\hline
\end{tabular}

fields per strip is given. The last column contains the overlap between fields in $\%$. By mirroring the grid along the equator one obtains a grid for the northern hemisphere. The combination of the grids for both hemispheres is a grid for the entire sky.

\section{References}

1. Begeman, K.G., Belikov, A.N., Boxhoorn, D.R., Valentijn, E.A.: The Astro-WISE paradigm. Experimental Astronomy Special Issue: Astro-WISE (in preparation, 2011)

2. Duda, R.O., Hart, P.E.: Use of the Hough transformation to detect lines and curves in pictures. Commun. ACM 15, 11 (1972)

3. Hough, P.V.C.: Machine analysis of bubble chamber pictures. In: Proc. Int. Conf. High Energy Accelerators and Instrumentation (1959)

4. McFarland, J.P., Helmich, E.M., Valentijn, E.A.: The Astro-WISE approach to quality control for astronomical data. Experimental Astronomy Special Issue: Astro-WISE (in preparation, 2011)

5. Mwebaze, J., Boxhoorn, D., Valentijn, E.: Astro-WISE: tracing and using lineage for scientific data processing. In: Proc. NBiS, 2009 International Conference on Network-Based Information Systems, p. 475 (2009)

6. Valentijn, E.A., McFarland, J.P., Snigula, J., Begeman, K.G., Boxhoorn, D.R., Rengelink, R., Helmich, E., Heraudeau, P., Kleijn, G.V., Vermeij, R., Vriend, W.-J., Tempelaar, M.J., Deul, E., Kuijken, K., Capaccioli, M., Silvotti, R., Bender, R., Neeser, M., Saglia, R., Bertin, E., Mellier, Y.: Astro-WISE: chaining to the universe. ASP Conf. Ser. 376, 491 (2007)

7. Valentijn, E.A., Kuijken, K., Kleijn, G.V.: OmegaCAM@VST. Experimental Astronomy Special Issue: Astro-WISE (in preparation, 2011)

8. Verdoes Kleijn, G., Vermeij, R., Valentijn, E., Kuijken, K.: The secondary standards programme for OmegaCAM. ASP Conf. Ser. 364, 103 (2007) 